\title{
Long Short Term Memory Network Using Grey Wolf Optimization for Stock Price Prediction
}

\author{
Pravinkumar M. Sonsare ${ }^{*}$, Praful R. Pardhi and Roshni S. Khedgaonkar ${ }^{3}$ \\ ${ }^{1,2}$ Shri Ramdeobaba College of Engineering and Management, Nagpur, India \\ ${ }^{3}$ Yeshwantrao Chavan College of Engineering, Nagpur, India
}

\section{ABSTRACT}

Stock market is backbone of nation's economy. Stable and improving stock market is very important for economy. Stock price prediction is one of the trending topics in data science for researcher. Scientist, analyst, traders are looking for efficient method of prediction of stock price. For profit many investors are keen to know the future of stock market. So, powerful prediction method is required for shareholder. Many methods are implemented using machine learning and deep learning techniques. In this work, we proposed a hybrid framework. This framework consists of Long Short Term Memory Network (LSTM) with a Grey Wolf Optimizer (GWO) which is utilized to estimate stock costs. This proposed framework would improve exactness of prediction of stock cost and helps the investors. We designed traditional LSTM and LSTM with GWO. The results of LSTM with GWO shows better result than LSTM.

\section{KEY WORDS: STOCK PRICE, RECURRENT NEURAL NETWORK, GREY WOLF OPTIMIZER, LONG SHORT TERM MEMORY NETWORK.}

\section{INTRODUCTION}

In computer science, "Prediction "is most difficult part to work. It is a goal to achieve for most of the researcher. Stock market is very important part of countries economy. So that prediction of stock is one of the topic in which many researcher wants to work. Many models are found for stock prediction. There are many factors available for prediction of stock. External macroeconomic factors are used to predict stock(Lin, Chu, \&t Wang, 2018).Value, quantity and financial facts are used by most of the researcher to predict cost of stock (B. Mendelsohn Louis, 2000).

\section{ARTICLE INFORMATION}

Received 20th Oct 2020 Accepted after revision 30th Dec 2020 Print ISSN: 0974-6455 Online ISSN: 2321-4007 CODEN: BBRCBA

Thomson Reuters ISI Web of Science Clarivate Analytics USA and Crossref Indexed Journal

\section{Clarivate
Analytics}

NAAS Journal Score 2020 (4.31) SJIF: 2020 (7.728)

A Society of Science and Nature Publication,

Bhopal India 2020. All rights reserved.

Online Contents Available at: http//www.bbrc.in/

Doi: http://dx.doi.org/10.21786/bbrc/13.14/13
Many statistical methods are used for testing market efficiency(SteveHogan,Robert Jarrow,MelvynTeo, 2003). Nowadays many machine learning algorithms are used to predict stock price. Support vector regression and principal component analysis are used to analyze share at London stock market(Fu \& Patra, 2009).Long short term memory network is deployed on S \& P 500 for prediction of financial market prediction task. This method is used to select profitable trading in market. Simplified ruled based trading strategy is devised for LSTM prediction(Fischer $\mathrm{Ct}$ Krauss, 2018).Hybrid training model consists of Artificial Neural Network and Genetic Algorithm to predict Thailand SET50 index trend.

This method is used on data collected from 2009 to 2014(Inthachot, Boonjing, Ct Intakosum, 2016).Artificial neural network is used to predict ISE index and Dow Jones index (E. Birgul, M. Ozturan, 2003)(J. Bollen, H. Mao, 2011). Trend deterministic data of technical indicators is proposed to train model. It showed better result than conventional methods. This imported data is used for various machine learning algorithm to analyze CNX Nifty and S\&P Bombay Stock Exchange markets(J.

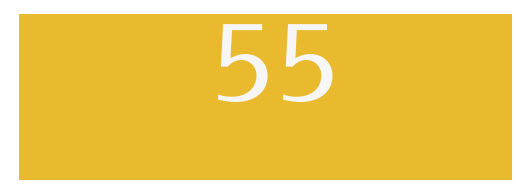


Patel, S. Shah, P. Thakkar, 2015).Stacked autoencoders with LSTM is used to forecast stock price(Bao, WeiYue, Jun, 2017).

Neural networks are used for faster convergence with the help of learning rates. The problem associated with neural network is to select appropriate learning rate. Fast or slow learning rate causes an erroneous result in linear or nonlinear network. Network undergoes many epochs to get optimal solution by reinitializing the weights(Sikder MF, Uddin MJ, 2016)(Sonsare \&t Gunavathi, 2019). The stochastic algorithms which are inspired from behavior of animal avoids problems occur in neural network with backpropagation. These algorithms used initial solution which will be optimized using various iterations. Our aim is to predict the future stock prices using the data from ' $N$ ' days. This dataset will be evaluated for better predictions using Machine Learning Techniques with Python to predict the future stock prices with high degree of accuracy. We designed long short term memory network with grey wolf optimizer to predict stock price.

Preliminaries: One of the challenging problems in data science is sequence prediction. This domain has wide range of problem in various fields like natural language processing, bioinformatics, stock market etc. The recent development in data science shows long short term memory (LSTM) network which gives most effective solution. This is because of network's ability to remember pattern of sequence for long duration of time. Due to this property of LSTM, it overcomes conventional neural network and recurrent neural network.

Recurrent Neural Network: Many traditional networks do not have memory to learn pattern in a sequence. Recurrent Neural Network is perfect solution to learn pattern in sequences. Data which has been learned by recurrent neural network are maintained by loop as shown in fig 2.1 .

Figure 2.1: Recurrent Neural Network (RNN)

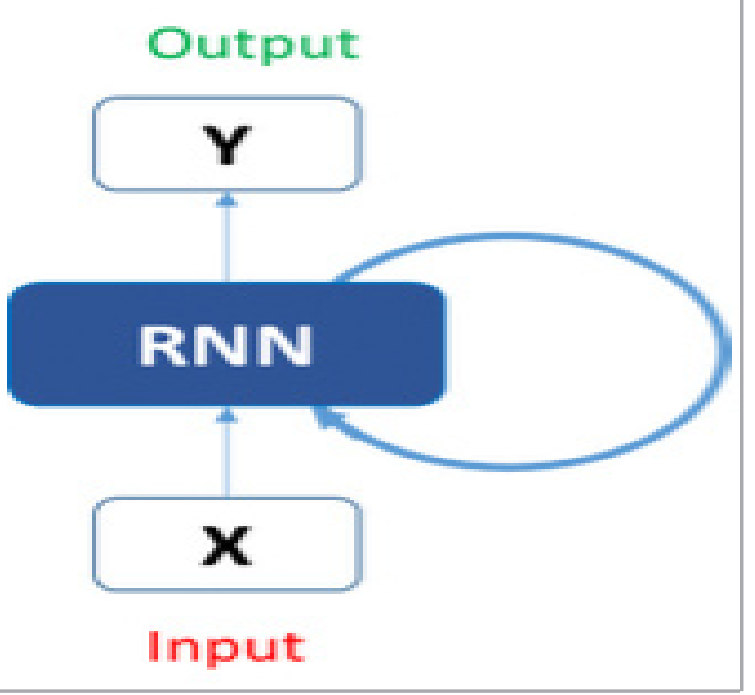

The RNN produce output based on iterations performs on input sequence over time. The RNN requires current time and current state which is previous output for generating output. The RNN perform transformation on input matrices using weight and bias matrices. The pseudocode of the RNN is as follows(CHOLLET, 2018). first_state $=0$

for sequence_t in stock_sequence:

sequence_t $=$ activation $(\operatorname{dot}(W$, sequence_t $)+\operatorname{dot}(U$, Initial_t) + bias)

initial_t $=$ secstr_t

RNN works effectively for sequence where there are short term dependencies. But RNN fails in long term dependencies. RNN does not consider context of sequence because of vanishing gradient problem. This problem makes training of layer difficult because small part of derivative multiplies error many times while reaching towards first layer.

Long Short term Memory Cell: The solution to avoid vanishing gradient problem is use of LSTM network. The LSTM cells are used to carry information along every timesteps. LSTM prevents older signals from gradually vanishing during the learning process. This saves information for future processing. In LSTM, data is carried across timesteps (Ct) which converts the current output to next state. LSTM experiences three distinct transformations in the form of RNN. All three transformations have different weight matrices. Pseudocode for RNN with LSTM as follows(CHOLLET, 2018).

secstr_t $=$ activation(dot(initial_t, Uo) $+\operatorname{dot}($ sequence_t, Wo) + dot(Carry_t, Vo) + bias)

$\mathrm{a} \_\mathrm{t}=\operatorname{activation}(\operatorname{dot}($ initial_t, Ui) $+\operatorname{dot}($ sequence_t, Wi) + biasa)

b_t $=$ activation(dot(initial_t, Uf) $+\operatorname{dot}($ sequence_t, Wf) + biasb)

c_t $=$ activation(dot(initial_t, Uk) $+\operatorname{dot}($ sequence_t, Wk) + biasc)

Next carry_t can be obtained by

carry_t $+1=a_{-} t * c_{-} t+$ carry_t ${ }^{*} b_{-} t$

Grey Wolf Optimization: Grey wolf optimizer is introduced by Mirjalili (Mirjalili, Mirjalili, \&t Lewis, 2014). This swarm based algorithm is inspired from hunting style of grey wolf. Grey wolves are divided into Alpha (a), Beta (ß), Delta (d), and Omega ( $\omega)$. Alpha (a), Beta ( $(3)$, Delta (d) are considered as finest fitting wolves and guide Omega $(\omega)$ to optimal search area. This optimization is guided by Alpha (a), Beta ( $(3)$, and Delta (d). The position of wolves changes around finest fitting wolves. The position of wolves may change relative to change in position of prey. The grey wolves are continuing hunting till prey does not stop moving. Omega's ( $\omega)$ updates it's position relative to Alpha (a), Beta ( $(3)$, Delta (d). Prey is moving in area which is encircled. The pseudo code of optimization process can be stated as follows(Mirjalili et al., 2014):

Consider Pi is the grey wolf population

Initialize the search agent position 
Each search agent's fitness is computed

SA1,SA2,SA3 are first ,second and third search agent respectively

Repeat until iteration reaches to maximum

for each search agent

Modify position and compute fitness

Increment iteration

End repetition

return finest search agent

Modified LSTM with GWO: The sequence is fed up to long short term memory cell for training. After numbers of iterations total mean square error (TMSE) is computed. This TMSE is passed to grey wolf optimizer. GWO assess TMSE around the fitness of Alpha (a), Beta ( $(3)$, and Delta (d). Best wolves represent the search agent. Best wolves are identified by its fitness and position. Weight and bias matrices indicates search agent's position. Position is adjusted iteratively. Adjustment is based on the number of search agents. In this way GWO updates the weights and biases. The updated weights and biases are finally passed to the LSTM.

Figure 2.2: LSTM with GWO

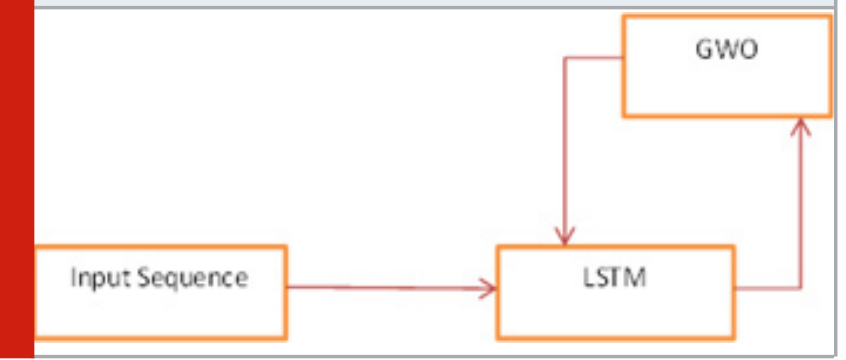

Proposed Methodology: We have processed NSE data from 01.01.2011 to 31.12.2016. We preprocessed dataset.
Figure 3.1: Proposed Methodology

Rav data collection

Data Preprocessing

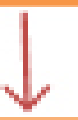

Feature Extraction

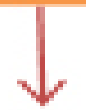

Neural Network Training

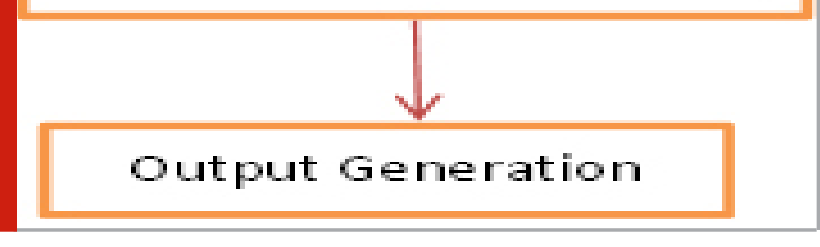

We perform discretization and normalization of data. Data cleaning is done by replacing the missing values. There are many features in dataset. We extracted few important features like date, open, high, low, close and volume. These features are fed up to neural network. We designed three layer long short term memory and one dense layer network. We train this model with initial bias and weight matrix. We used grey wolf optimizer to update weights and sigmoid activation function for dense output layer.

Table 1. Comparison of LSTM with and without GWO

\begin{tabular}{|l|c|c|c|c|c|}
\hline \multirow{2}{*}{ Features } & \multirow{2}{*}{$\begin{array}{c}\text { No of } \\
\text { iterations }\end{array}$} & \multicolumn{2}{|c|}{ LSTM } & \multicolumn{2}{c|}{ LSTM with GWO } \\
\cline { 3 - 6 } & & Train RMSE & Test RMSE & Train RMSE & Test RMSE \\
\hline O/C & 100 & 0.032 & 0.029 & 0.021 & 0.019 \\
\hline O/C & 200 & 0.030 & 0.028 & 0.014 & 0.012 \\
\hline O/C & 300 & 0.027 & 0.025 & 0.010 & 0.006 \\
\hline H/L & 100 & 0.028 & 0.025 & 0.022 & 0.020 \\
\hline H/L & 200 & 0.026 & 0.024 & 0.020 & 0.018 \\
\hline H/L & 300 & 0.025 & 0.022 & 0.018 & 0.015 \\
\hline H/L/C & 100 & 0.022 & 0.019 & 0.013 & 0.011 \\
\hline H/L/C & 200 & 0.019 & 0.017 & 0.008 & 0.007 \\
\hline H/L/C & 300 & 0.016 & 0.013 & 0.005 & 0.004 \\
\hline
\end{tabular}

\section{RESULTS AND DISCUSSION}

We used 1312 sequence from dataset. Out of these sequences we have used 1180 samples for training and 132 samples for testing. We have used different set of parameter to measure the efficiency of model. We checked the efficiency of model using root mean square method. Root mean square error is square root of sum of all error. We used python for training with Keras as frontend and Tensorflow as backend. We used features like open-close (O/C), high-low (H/L), high-low-close $(\mathrm{H} / \mathrm{L} / \mathrm{C})$ We used LSTM and LSTM with grey wolf optimizer for training 
model. The result of model using different parameter set and epochs shown in Table1.

We have modeled LSTM and LSTM with Grey wolf parameter. We used these models for feature set like Open/Close, High/Low and High/Low/Close. We observed that LSTM with GWO gives better result than traditional LSTM. We also found that feature set High/Low/Close is an optimized set of features to produce better result.

\section{CONCLUSION}

Nowadays researchers are encouraged for prediction of stock price. This is because of growing popularity of stock market. These prediction methods are helpful for investors to play in stock market as well as researcher to predict stock price more accurately. In this regard we devised method consists of LSTM with GWO. This method will be helpful for brokers, researchers or any person who want to play stock market. This method may help investors to know future situation of stock market.

\section{REFERENCES}

B. Mendelsohn Louis. (2000). Trend Forecasting with Technical Analysis: Unleashing the Hidden Power of Intermarket Analysis to Beat the Market.

Bao, WeiYue, Jun, Y. R. (2017). A deep learning framework for financial time series using stacked autoencoders and longshort term memory. PLoS ONE. CHOLLET, F. (2018). Deep Learning with Python.

E. Birgul, M. Ozturan, and B. B. (2003). Stock market prediction using artificial neural networks. Proceedings of the in 3rd Hawaii International Conference on Business.

Fischer, T., \& Krauss, C. (2018). Deep learning with long short-term memory networks for financial market predictions. European Journal of Operational Research, 270(2), 654-669. https://doi.org/10.1016/j. ejor.2017.11.054

Fu, X., \&t Patra, A. (2009). Machine Learning in Statistical Arbitrage. Retrieved from http://cs229. stanford.edu/proj2009/FuPatra.pdf

Inthachot, M., Boonjing, V., \&t Intakosum, S. (2016). Artificial Neural Network and Genetic Algorithm Hybrid Intelligence for Predicting Thai Stock Price Index Trend. Computational Intelligence and Neuroscience, 2016. https://doi.org/10.1155/2016/3045254

J. Bollen, H. Mao, and X. Z. (2011). Twitter mood predicts the stock market. Journal of Computational Science, 2(1), 1-8.

J. Patel, S. Shah, P. Thakkar, and K. K. (2015). Predicting stock and stock price index movement using Trend. Expert Systems with Applications, 42(1), 259-268.

Lin, B. S., Chu, W. T., \& Wang, C. M. (2018). Application of Stock Analysis Using Deep Learning. Proceedings 2018 7th International Congress on Advanced Applied Informatics, IIAI-AAI 2018, 612-617. https://doi. org/10.1109/IIAI-AAI.2018.00128

Mirjalili, S., Mirjalili, S. M., \&t Lewis, A. (2014). Grey Wolf Optimizer. Advances in Engineering Software, 69, 4661. https://doi.org/10.1016/j.advengsoft.2013.12.007

Sikder MF, Uddin MJ, H. S. (2016). Predicting students yearly performance using neural network: A case study of BSMRSTU. InInformatics, Electronics and Vision (ICIEV), 2016 5th International Conference, 524-529.

Sonsare, P. M., \&t Gunavathi, C. (2019). Investigation of machine learning techniques on proteomics: A comprehensive survey. Progress in Biophysics and Molecular Biology, 149. https://doi.org/10.1016/j. pbiomolbio.2019.09.004

SteveHogan,Robert Jarrow,MelvynTeo, M. (2003). Testing market efficiency using statistical arbitrage with applications to momentum and value strategies. Journal of Financial Economics, 73(3), 525-565. 